\title{
A Diagnostic System for Speed-Varying Motor Rotary Faults
}

\author{
Chwan-Lu Tseng, Shun-Yuan Wang, Shou-Chuang Lin, Jen-Hsiang Chou, and Ke-Fan Chen
}

Department of Electrical Engineering, National Taipei University of Technology, Taipei 10608, Taiwan

Correspondence should be addressed to Shun-Yuan Wang; sywang@ntut.edu.tw

Received 26 February 2014; Accepted 18 April 2014; Published 19 May 2014

Academic Editor: Her-Terng Yau

Copyright (C) 2014 Chwan-Lu Tseng et al. This is an open access article distributed under the Creative Commons Attribution License, which permits unrestricted use, distribution, and reproduction in any medium, provided the original work is properly cited.

\begin{abstract}
This study proposed an intelligent rotary fault diagnostic system for motors. A sensorless rotational speed detection method and an improved dynamic structural neural network are used. Moreover, to increase the convergence speed of training, a terminal attractor method and a hybrid discriminant analysis are also adopted. The proposed method can be employed to detect the rotary frequencies of motors with varying speeds and can enhance the discrimination of motor faults. To conduct the experiments, this study used wireless sensor nodes to transmit vibration data and employed MATLAB to write codes for functional modules, including the signal processing, sensorless rotational speed estimation, neural network, and stochastic process control chart. Additionally, Visual Basic software was used to create an integrated human-machine interface. The experimental results regarding the test of equipment faults indicated that the proposed novel diagnostic system can effectively estimate rotational speeds and provide superior ability of motor fault discrimination with fast training convergence.
\end{abstract}

\section{Introduction}

The abnormal vibrations observed during motor rotary faults are typically caused by incorrect shaft connection or friction between rotary components [1-4]. Traditionally, maintenance personnel had to shut down motors for examination and maintenance. However, the vibration detection method, which involves simply attaching an accelerometer to the motor $[5,6]$ for analysis and measurement, fulfills the demand for real-time, online, and minimally invasive diagnosis.

For experiments regarding vibration faults, time-domain signals are typically converted into frequency-domain signals before analysis. For example, Iorgulescu et al. [7] employed a fast Fourier transform (FFT) to achieve this objective, and Garcia-Perez et al. [8] adopted power spectral density (PSD) to complete a conversion to frequency-domain signals for further analysis.

Previous studies of motor faults have typically focused on measurements of single rotational speeds; however, changes in rotational speed alter fault characteristic frequencies, resulting in incorrect diagnosis. In recent years, scholars have begun considering variations in rotational speeds when diagnosing faults. For instance, Teotrakool et al. [9] used wavelet packet decomposition to diagnose bearing faults under various rotational speeds. Arabaci and Bilgin [10] employed torque speed curves to diagnose rotor bar broken in motors. Also, Blodt et al. [11] conducted a time-frequency analysis to detect torque vibrations under various rotational speeds. These studies further adopted a motor current signature analysis to diagnose motor faults with various rotary frequencies.

However, these studies required the installation of tachometers and the a priori input of rotary frequencies or rotational speeds into diagnostic systems. Inspired by the research done by Ocak and Loparo [12], who employed the physical characteristics of frequency spectra, this study proposed a sensorless method that enables a system to automatically detect the motor rotational speed, thereby reducing the tachometer installation cost.

The development of neural networks in modern intelligent motor fault diagnostic systems is quite mature [13, 14]. Such networks possess substantial parallel computing abilities, adaptability, fault tolerance, self-learning abilities, 
and memory abilities and are, hence, suitable for diagnosing motor faults. The disadvantages of traditional neural networks are that the structure such as the appropriate number of neurons is difficult to know and the training is time consuming. To solve the neuron choice problem, Chuang et al. [15] proposed a dynamic structural neural network (DSNN) that was applied to power quality classification. Still, the training of DSNN is time consuming.

In addition to the above mentioned considerations, this study also incorporated hybrid discriminant analysis (HDA). HDA is a combination of principal component analysis (PCA) and discriminant analysis (DA). These two analysis methods are commonly used in statistics, medicine, and pattern recognition to categorize data and reduce data dimensionality. Researchers $[16,17]$, for example, used PCA in a motor fault analysis and Ayhan et al. [18] combined DA with a neural network for diagnosing the faults caused by rotor bar damage. HDA, which is based on PCA and DA, can be used to identify optimal data classification results. Converted data are then fed into a neural network to increase the speed of learning and discrimination during training.

In this study, motor vibration signals are firstly measured. Subsequently, the vibrational time-domain signals are converted into frequency-domain signals using the fast Fourier transform (FFT) algorithm. The sensorless rotational speed estimation method was used to obtain the current rotatory frequency and rotational speed from spectrograms as a reference for extracting motor fault characteristics. Also, a normalization procedure was used to preprocess the spectral characteristics. To speed up the convergence of training dynamic structural neural network, a hybrid discriminant analysis (HDA) was used to reduce the influence of insignificant characteristics. Different from the conventional motor diagnostic system, this study used the improved dynamic structural network with the terminal attractor method [19] to update the weights (hereafter, called HTAIDSNN) for fast convergence and proposed a novel diagnostic system which is capable of detecting rotary faults in motors at different motor speeds. Furthermore, in practice, to eliminate the influence of noises or outliers, this study also established a dual core diagnostic system that comprised the HTAIDSNN and statistical process control (SPC) techniques [20].

\section{Vibration Characteristics of Motor Rotary Faults and the Normalization}

Mechanical vibrations during the operation of machines are among the main features of equipment damage or deterioration. When a motor experiences a fault, different types of motor faults will correspond with different fault characteristics on the spectrogram. Spectrograms are composed of a specific motor rotary frequency $f_{r}$ and frequency multipliers, in which different fault types show different peak values for each multiplier. For AC motors, the synchronous speed, rotational speed, and rotary frequencies can be obtained using

$$
N_{s}=\frac{120 \times f_{s}}{P}
$$

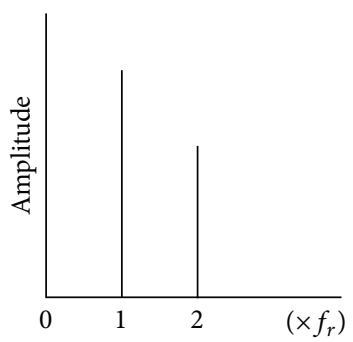

(a)

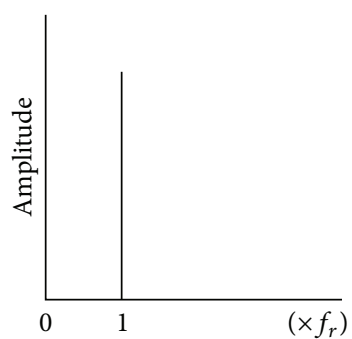

(b)

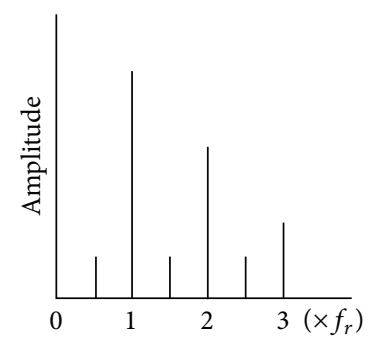

(c)

FIGURE 1: Spectral characteristics: (a) bent shafts; (b) imbalanced rotors; and (c) loose screw components.

$$
\begin{gathered}
N_{r}=(1-S) \times N_{s} \\
f_{r}=\frac{N_{r}}{60},
\end{gathered}
$$

where $N_{s}$ represents the synchronous speed of the motors, $f_{s}$ represents the supply frequency, $P$ represents the number of poles, $S$ represents the slip rate, $N_{r}$ represents the actual speed, and $f_{r}$ represents the rotary frequency.

After obtaining the rotary frequency of a motor, motor fault can be diagnosed using the rotary frequency and amplitudes related to frequency multipliers. Three most common types of motor rotary faults are bent shafts, imbalanced rotors, and loose screw components. The spectral characteristics of these types of faults are depicted in Figure 1. As indicated in Figure 1, different types of faults correspond to different characteristic frequency spectra; thus, the motor fault type can be determined by examining the specific pattern of a frequency spectrum.

The corresponding spectral characteristics examined during motor fault diagnoses are called fault characteristic parameters. Equation (2) is the mathematical notion of the types of faults, fault characteristics, and fault characteristic parameters

$$
F(n)=C\left(a_{n, 1}, a_{n, 2}, a_{n, 3}, \ldots, a_{n, k}, \ldots, a_{n, K}\right),
$$

where $F(n)$ denotes the fault type, $C$ represents the fault characteristics, $a_{n, 1}, a_{n, 2}, \ldots, a_{n, k}, \ldots$, and $a_{n, k}$ represent the fault characteristic parameters of the $n$ th-segment signal, $k$ represents the $k$ th characteristic frequency, and $K$ denotes the number of characteristic frequencies.

When faults occur, the amplitudes of specific frequency multipliers change accordingly. The fault characteristic parameters used in this study are defined as $0.5 f_{r}$, 
$1 f_{r}, 1.5 f_{r}, 2 f_{r}, 2.5 f_{r}, 3 f_{r}$, and $3.5 f_{r}$. As shown in (3), a vibration eigen-matrix $H$ of a healthy motor is compiled using fault characteristics based on the $n$-segment vibrational data collected from healthy motors collected in this study:

$$
H=\left[\begin{array}{cccc}
h_{1,1} & h_{1,2} & \cdots & h_{1, K} \\
h_{2,1} & h_{2,2} & \cdots & h_{2, K} \\
\vdots & \vdots & \ddots & \vdots \\
h_{n, 1} & h_{n, 2} & \cdots & h_{n, K}
\end{array}\right]
$$

The means and standard deviations of amplitudes of these characteristic frequencies are then calculated by using

$$
\begin{gathered}
H_{\text {mean value }}[k]=\frac{1}{n} \sum_{q=1}^{n} h_{q, k}, \\
H_{\text {standard deviation }}[k]=\sqrt{\frac{1}{n} \sum_{q=1}^{n}\left(h_{q, k}-H_{\text {mean value }}[k]\right)^{2}} .
\end{gathered}
$$

The amplitude of each characteristic frequency of the $n$ segment signal is normalized by

$$
\begin{gathered}
h_{z_{q, k}}=\frac{h_{q, k}-H_{\text {mean value }}[k]}{H_{\text {standard deviation }}[k]}, \\
H_{z}=\left[\begin{array}{cccc}
h_{z_{1,1}} & h_{z_{1,2}} & \cdots & h_{z_{1, K}} \\
h_{z_{2,1}} & h_{z_{2,2}} & \cdots & h_{z_{2, K}} \\
\vdots & \vdots & \ddots & \vdots \\
h_{z_{n, 1}} & h_{z_{n, 2}} & \cdots & h_{z_{n, K}}
\end{array}\right] .
\end{gathered}
$$

When normalizing the vibration eigen-matrices of different fault types, the amplitude means and standard deviations of the characteristic frequencies for healthy motors are employed as the basis of normalization. Thus, all the normalization processes are performed using the same basis. As shown in (8), (9), and (10), the normalized fault eigenmatrices $B_{z}, I_{z}$, and $L_{z}$ are obtained by normalizing the fault eigen-matrices of bent shafts, imbalanced rotors, and loose components, respectively. The compiled normalized fault eigen-matrices are then used as training data for the neural networks. Fault eigen-matrices with a greater number of segments provide more training data, thereby increasing the discrimination ability of the networks. Consider the following:

$$
B_{z}=\left[\begin{array}{cccc}
b_{z_{1,1}} & b_{z_{1,2}} & \cdots & b_{z_{1, K}} \\
b_{z_{2,1}} & b_{z_{2,2}} & \cdots & b_{z_{2, K}} \\
\vdots & \vdots & \ddots & \vdots \\
b_{z_{n, 1}} & b_{z_{n, 2}} & \cdots & b_{z_{n, K}}
\end{array}\right]
$$

$$
\begin{gathered}
I_{z}=\left[\begin{array}{cccc}
i_{z_{1,1}} & i_{z_{1,2}} & \cdots & i_{z_{1, K}} \\
i_{z_{2,1}} & i_{z_{2,2}} & \cdots & i_{z_{2, K}} \\
\vdots & \vdots & \ddots & \vdots \\
i_{z_{n, 1}} & i_{z_{n, 2}} & \cdots & i_{z_{n, K}}
\end{array}\right], \\
L_{z}=\left[\begin{array}{cccc}
l_{z_{1,1}} & l_{z_{1,2}} & \cdots & l_{z_{1, K}} \\
l_{z_{2,1}} & l_{z_{2,2}} & \cdots & l_{z_{2, K}} \\
\vdots & \vdots & \ddots & \vdots \\
l_{z_{n, 1}} & l_{z_{n, 2}} & \cdots & l_{z_{n, K}}
\end{array}\right] .
\end{gathered}
$$

\section{Sensorless Rotational Speed Estimation}

Traditionally, an additional tachometer or an encoder is employed for measuring motor rotational speed. To reduce the measurement cost, this study proposes a sensorless rotational speed estimation method that determined motor rotational speed by analyzing the vibrational frequencydomain signals of motors.

Previous literature [12] has indicated that the harmonics of rotary frequency exhibit greater amplitudes in the vibration frequency spectra of motors. Considering this finding, this study first identifies the local maximums in relevant spectrograms and their corresponding frequencies. After identifying the frequencies that corresponded to the local maximums, an indicator $\alpha$ with an initial value of 0 is assigned to each frequency shown in Figure 2. If the corresponding frequency for one of the local maximums has a multiplicative relationship with a larger frequency, 1 is added to $\alpha$. If two relationships are determined, 2 is added to the $\alpha$ value, and so on. Because the actual frequency values do not exhibit exact multiplicative relationships, errors less than $3 \mathrm{~Hz}$ are allowed in this study. Finally, the frequency corresponding to the greatest $\alpha(\alpha=2)$ is selected as the estimated rotatory frequency. For example, a frequency of $30 \mathrm{~Hz}$ (the corresponding rotational speed is $1800 \mathrm{rpm}$ ) is lower than the frequencies of $43.8,60.7$, and $120.2 \mathrm{~Hz}$, with the frequencies of 60.7 and $120.2 \mathrm{~Hz}$ being two and four times (second and fourth harmonic generation) higher than that of $30 \mathrm{~Hz}$; thus, $\alpha$ is set as 2 . If the frequency is $60.7 \mathrm{~Hz}$, $120.2 \mathrm{~Hz}$ is a frequency that doubles the original value (second harmonic); therefore, $\alpha$ is set as 1 . The frequency with the greatest $\alpha$ is selected as the desired rotatory frequency $f_{r}$ as indicated in Figure 3.

To verify the speed estimation method using the above mentioned concept, a four-pole, one-hp, three-phase induction motor manufactured by the TECO Electric \& Machinery Company (Taiwan) is adopted for this experiment. The experiment performed ten times of measurement and estimation for rotational speeds from $900 \mathrm{rpm}$ to $1800 \mathrm{rpm}$. Table 1 summarizes the statistics of the estimated motor rotational speeds. The standard deviations are smaller than $4 \mathrm{rpm}$ for each rotational speed. Moreover, the estimation errors of rotational speeds are smaller than $2 \mathrm{rpm}$ and thus the accuracy of the proposed sensorless rotational speed estimation method is confirmed. 


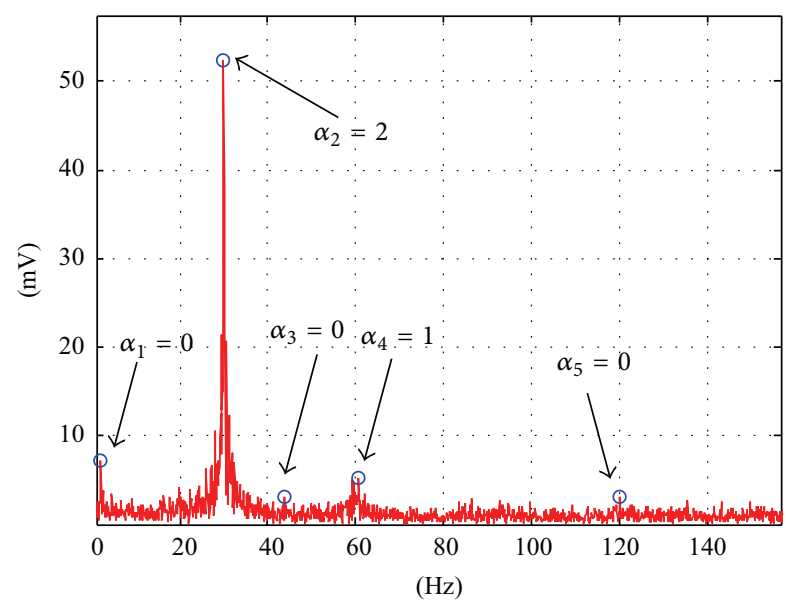

FIGURE 2: Indicator $\alpha$ for local maximums.

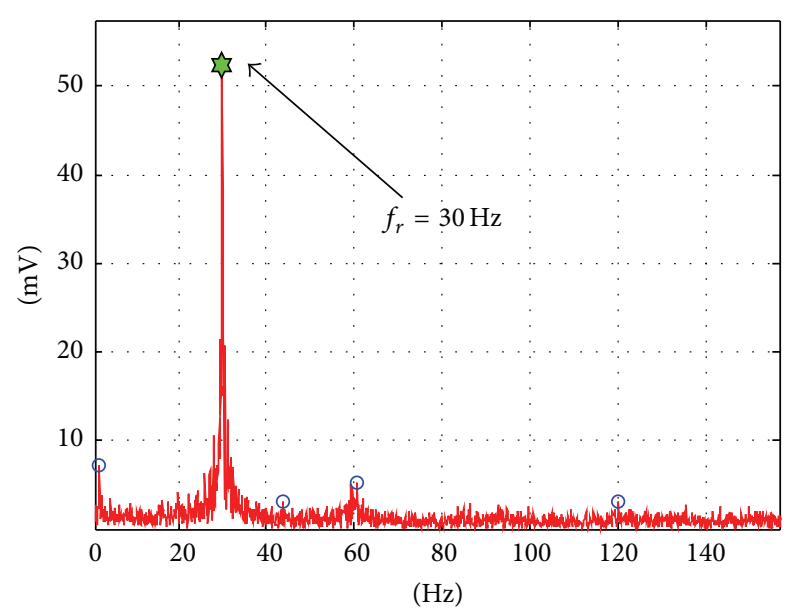

FIGURE 3: Rotary frequencies obtained using the estimation method.

TABLE 1: Statistics of the estimated motor rotational speeds.

\begin{tabular}{lcccc}
\hline $\begin{array}{l}\text { Specified } \\
\text { rotational } \\
\text { speed } \\
(\mathrm{rpm})\end{array}$ & $\begin{array}{c}\text { Average } \\
\text { measured } \\
\text { speed } \\
\left(f_{r}, \mathrm{rpm}\right)\end{array}$ & $\begin{array}{c}\text { Average } \\
\text { estimated } \\
\text { speed } \\
\left(f_{r e}, \mathrm{rpm}\right)\end{array}$ & $\begin{array}{c}\text { Standard } \\
\text { deviation } \\
\text { of } f_{r e}\end{array}$ & $\begin{array}{c}\text { Average } \\
\text { estimation } \\
\text { error } \\
f_{r e}-f_{r}\end{array}$ \\
\hline 900 & 899.55 & 899.90 & 3.41 & 0.45 \\
1000 & 1000.30 & 1001.90 & 3.52 & 1.60 \\
1200 & 1199.56 & 1200.10 & 2.94 & 0.54 \\
1350 & 1348.44 & 1348.80 & 2.87 & 0.36 \\
1400 & 1398.20 & 1398.30 & 2.83 & 0.10 \\
1600 & 1599.31 & 1600.40 & 3.55 & 1.09 \\
1800 & 1799.52 & 1800.00 & 2.82 & 0.48 \\
\hline
\end{tabular}

\section{The Improved Dynamic Structural Neural Network with the Hybrid Discriminant Analysis}

4.1. Improved Dynamic Structural Neural Network. In 2005, Chuang et al. proposed a DSNN capable of self-adjusting the number of neurons in the hidden layer [15]. Figure 4 shows a schematic diagram of the DSNN framework on a pseudo3D plane. The network is capable of adjusting the number of neurons in the hidden layer, which can be calculated using the following equation:

$$
N_{h}=x \cdot(y+p)(z+p)
$$

where $N_{h}$ represents the number of neurons in the hidden layer, $x$ represents the length of the hidden layer, $y$ represents the height of the hidden layer, $z$ represents the width of the hidden layer, and $p$ represents the number to which the neurons can be expanded, which can prevent neurons from increasing without limitation. Assume that there are $M$ neurons in the input layer, $N$ neurons in the output layer, and $H_{l}$ neurons in the $l$ th hidden layer, for $l=1,2, \ldots, k$. The total connection in Figure 4 indicates that all neurons in hidden layer are interconnected. Equation (12) shows the output vectors of the neurons in the first hidden layer. The output vectors for neurons in the other hidden layers are presented in (13), and the output vectors of the output layer are expressed in (14) as follows:

$$
\begin{gathered}
o_{h_{1}}=f\left[\left(\sum_{m}^{M} w_{h_{1}, m} x_{m}\right)-\theta_{h_{1}}\right], \\
o_{h_{j}}=f\left[\left(\sum_{i=1}^{j-1} \sum_{h_{i}}^{H_{i}} w_{h_{j}, h_{i}} o_{h_{i}}\right)-\theta_{h_{j}}\right], \quad j=2,3, \ldots, k . \\
o_{n}=f\left[\left(\sum_{h_{k}}^{H_{k}} w_{n, h_{k}} o_{h_{k}}\right)-\theta_{n}\right],
\end{gathered}
$$

where $o_{h_{1}}, o_{h_{j}}$, and $o_{n}$ are the outputs of the neurons in the first and $j$ th hidden layer and the output layer, respectively. $f[\cdot]$ is the activation function; $w_{h_{1}, m}$ is the weight value connecting the input layer and the first hidden layer neurons; $w_{h_{j}, h_{i}}$ is the weight value connecting the $i$ th and $j$ th hidden layer neurons; $w_{n, h_{k}}$ is the weight value connecting the $k$ th hidden layer and the output layer neurons; and $\theta_{h_{1}}, \theta_{h_{j}}$, and $\theta_{n}$ are the threshold or bias values of the neurons in the first and $j$ th hidden layer and the output layer. In (12), (13), and (14), the arguments of all variables are expressed as $m=1,2,3, \ldots, M$, $h_{1}=1,2,3, \ldots, H_{1}, h_{i}=1,2,3, \ldots, H_{i}, h_{j}=1,2,3, \ldots, H_{j}$, and $n=1,2,3, \ldots, N$, representing the numbers of neurons in the respective layers.

4.2. The Terminal Attractor Method. The error function is defined as (15), where $d_{n}$ is the expected output and $o_{n}$ is the actual output vector. The number of neurons in the output layer is represented by $n=1,2, \ldots, N$. Therefore,

$$
E=\frac{1}{2} \sum_{n=1}^{N}\left(d_{n}-o_{n}\right)^{2} .
$$

During the learning process for a neural network, the steepest descent method is the option most commonly employed for reducing the error function in (15) to a minimum. However, this method tends to constrain the function 


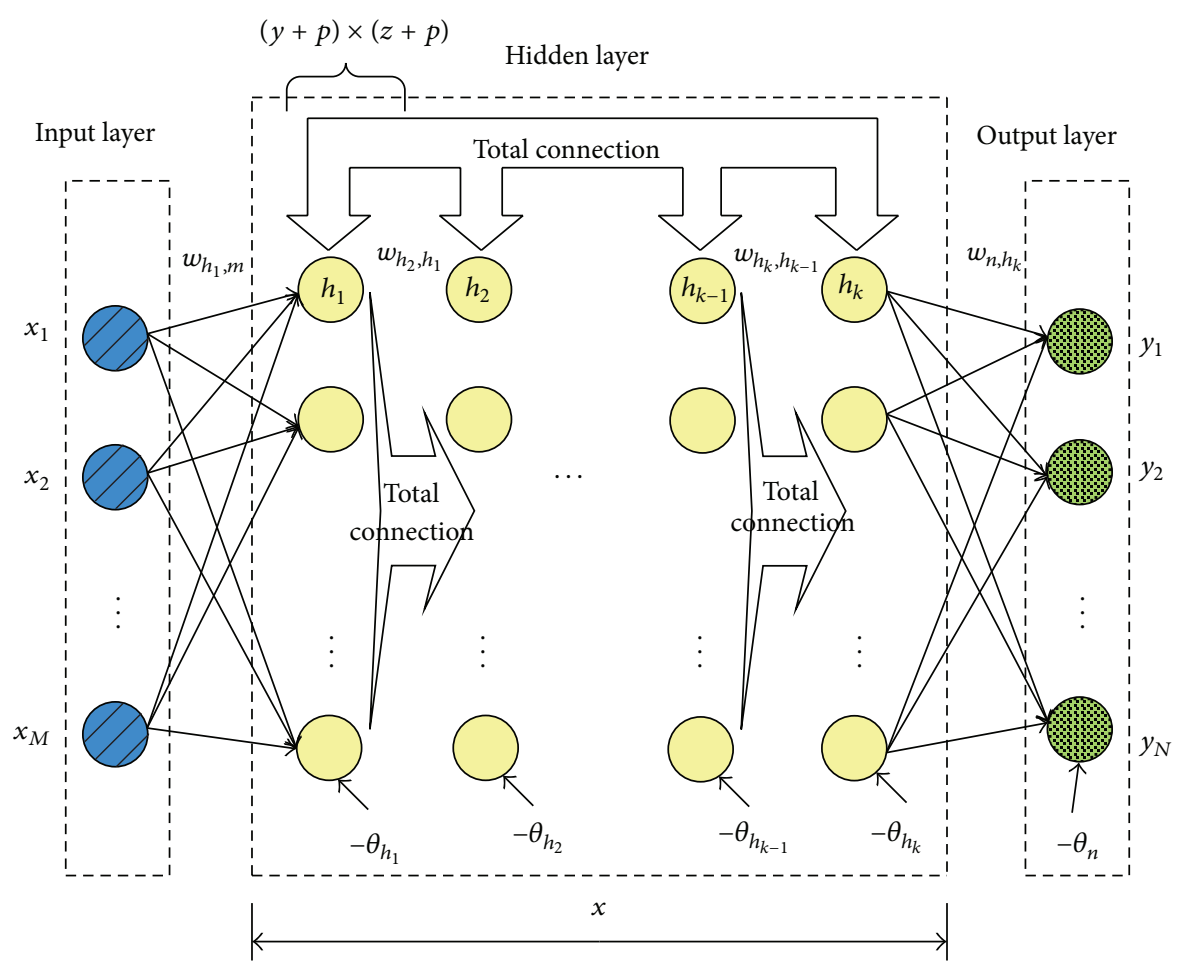

Figure 4: Pseudo-3D schematic of the HTAIDSNN hidden layers.

in a local optimal solution. This leads to the fact that the error cannot converge to zero within a limited time. To resolve this issue, Wang and Hsu incorporated heuristic terminal attractors into back-propagation neural networks in 1991 [19]. Assume that $w$ is the aggregated weight vector and let the derivative of $w$ satisfy

$$
\frac{d w}{d t}=-\gamma \Delta w, \quad \Delta w=\frac{\partial E}{\partial w}, \gamma=\frac{E^{k}}{\|\Delta w\|^{2}},
$$

where $0<k<1$. Consequently, the derivative of error function becomes

$$
\frac{d E}{d t}=-(\Delta w)^{T}\left(\frac{E^{k}}{\|\Delta w\|^{2}} \cdot \Delta w\right)=-E^{k}
$$

which determines a terminal attractor. Using the concept, the update law of the weight vector in this paper is given as

$$
\begin{gathered}
w(n+1)=w(n)+\mu \gamma(n) \Delta w(n), \quad \text { if } \gamma>1, \\
w(n+1)=w(n)+\mu \Delta w(n), \quad \text { if } \gamma<1,
\end{gathered}
$$

where $\mu$ is the learning rate. Hereafter, the proposed improved dynamic structural neural network is abbreviated as HTAIDSNN (heuristic terminal attractor improved dynamic system neural networks) and the learning rate $\mu$ is set to 0.9 .
4.3. Applying Hybrid Determinant Analysis to HTAIDSNN. To identify the eigen-matrix of principal components, $X^{(j)}$, which possesses various classes of data, is considered as

$$
X^{(j)}=\left[\begin{array}{cccc}
x_{11}^{(j)} & x_{12}^{(j)} & \cdots & x_{1 N_{j}}^{(j)} \\
x_{21}^{(j)} & x_{22}^{(j)} & \cdots & x_{2 N_{j}}^{(j)} \\
\vdots & \vdots & \ddots & \vdots \\
x_{k 1}^{(j)} & x_{k 2}^{(j)} & \cdots & x_{k N_{j}}^{(j)}
\end{array}\right],
$$

where $j$ denotes the class number in sequence, $N_{j}$ denotes the times of collection in the $j$ th class, $k$ denotes the number of factors and characteristics considered, and $x_{k N_{j}}^{(j)}$ denotes the value of the $k$ th characteristic at the $N_{j}$ th time under the $j$ th class. Let $x_{i}^{(j)}=\left[x_{1 i}^{(j)}, x_{2 i}^{(j)}, \ldots, x_{k i}^{(j)}\right]^{T}$, which represents the eigenvector collected in the ith time under the $j$ th class, and let $i=1,2, \ldots, N_{j}$. The average of the characteristics in each class is described using (20), where $m_{j} \in \mathfrak{R}^{k}$ and $C$ represents the number of classes considered. The average of all class characteristics is shown in (21), where $m \in \mathfrak{R}^{k}$ :

$$
\begin{gathered}
m_{j}=\frac{\sum_{i=1}^{N_{j}} x_{i}^{(j)}}{N_{j}}, \\
m=\frac{\sum_{j=1}^{C} N_{j} \cdot m_{j}}{\sum_{j=1}^{C} N_{j}} .
\end{gathered}
$$

The covariance matrix for the data is presented using (22), where $T$ represents the transpose or transposition symbol. 
Finally, through (23), the transformation matrix $W_{\mathrm{PCA}}$ is obtained following PCA:

$$
\begin{gathered}
S=\frac{1}{C} \sum_{j=1}^{C} \frac{1}{N_{j}} \sum_{i=1}^{N_{j}}\left(x_{i}^{(j)}-m\right)\left(x_{i}^{(j)}-m\right)^{T}, \\
W_{\text {PCA }}=\arg \max _{W} \operatorname{det}\left(W^{T} S W\right) .
\end{gathered}
$$

Using (19), (20), and (21), this study independently establishes matrices for within-class variance (24) and betweenclass variance (25). The ratio of $S_{B}$ to $S_{W}$ is larger, and the discrimination is better. Finally, using the Fisher DA method, the transformation matrix $W_{\mathrm{FDA}}$ is obtained by using (26)

$$
\begin{gathered}
S_{W}=\sum_{j=1}^{C} \sum_{i=1}^{N_{j}}\left(x_{i}^{(j)}-m_{j}\right)\left(x_{i}^{(j)}-m_{j}\right)^{T}, \\
S_{B}=\sum_{j=1}^{C} N_{j} \cdot\left(m_{j}-m\right)\left(m_{j}-m\right)^{T}, \\
W_{\mathrm{FDA}}=\arg \max _{W} \frac{\operatorname{det}\left(W^{T} S_{B} W\right)}{\operatorname{det}\left(W^{T} S_{W} W\right)} .
\end{gathered}
$$

Although between-class variances can increase during performing PCA, within-class variances also increase. Therefore, DA appears to be a more appropriate method for relevant computations. However, for data classes with a limited sample size or uneven sample distribution, PCA is superior to DA. To enhance the discrimination of data analysis, HDA [20] is used to obtain the optimal transformation matrix for given data. Equation (27) shows the transformation matrix of HDA:

$$
\left(W_{\mathrm{HDA}}, \lambda^{*}, \eta^{*}\right)=\arg \max _{W, \lambda, \eta} \frac{\operatorname{det}\left(W^{T}\left[(1-\lambda) \cdot S_{B}+\lambda \cdot S\right] W\right)}{\operatorname{det}\left(W^{T}\left[(1-\eta) \cdot S_{W}+\eta \cdot I\right] W\right)},
$$

where $I$ represents the identity matrix and $\lambda$ and $\eta$ represent parameters that are adjustable between 0 and 1 . Beginning at $\lambda=0$ and $\eta=0$, the parameters can increase by 0.1 to obtain the optimal HDA transformation matrix $W_{\mathrm{HDA}}$. Equation (28) shows the new matrix HDA_ $X^{(j)}$ obtained after the transformation:

$$
\mathrm{HDA}_{X}^{(j)}=W_{\mathrm{HDA}}^{T} \cdot X^{(j)}
$$

To perform the HDA, firstly the seven frequencymultiplier amplitudes considered in Section 2 are measured 20 times for various motor faults. The normalized fault eigenmatrices are then compiled using (7), (8), (9), and (10). These matrices are substituted into (19), where the total number of classes $j$ is 4 and the times or frequency for every class $N_{j}$ is equal to 20. The covariance matrix $S$, within-class variance matrix $S_{W}$, and between-class variance matrix $S_{B}$ are obtained by substituting them into HDA. The adjustable parameters $\lambda$ and $\eta$ are set to 0.4 and 0.7 , respectively. Finally, a new fault eigen-matrix is obtained with (28). Generally, when using hybrid determinants or principal components,

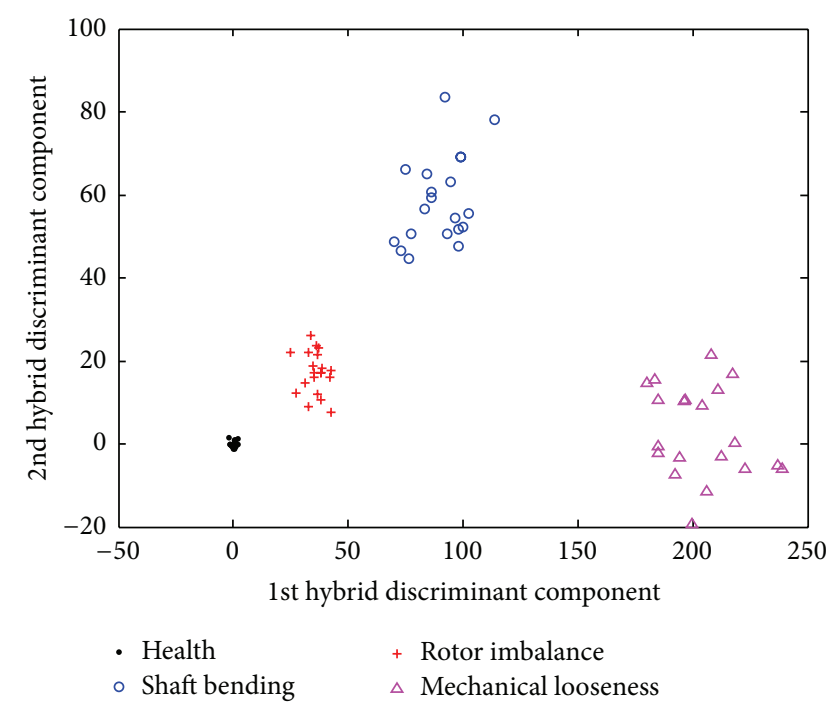

FIGURE 5: Distribution of the first two hybrid discriminant components.

the distribution of the first two components is analyzed [20], because the components are the most suitable for representing data traits. Figure 5 shows the distribution of the first two hybrid discriminant components.

Figure 5 shows that HDA provides comprehensive data classification and small within-class distance or variance and enhanced data discrimination. When clustering or grouping for fault classification is used, the relationship between only two component values is typically considered. This study combined the HTAIDSNN with the fault eigen-matrices converted by HDA. Based on the compilation of the fault eigenmatrices, this study established a neural network training matrix, as shown in Figure 6, where $\mathrm{hd}_{i}$ denotes the $i$ th hybrid discriminant or discrimination and $i=1,2, \ldots, 7 . \mathrm{hd}_{i}$ is used as the input neuron value. Regarding the parameters of neural networks, the HTAIDSNN possessed seven neurons in the input layer and four neurons in the output layer. Equation (11) shows the framework parameters of the hidden layer, where $x=4, y=5$, and $z=2$. Furthermore, the number to which the neurons could be expanded is $p=2$; therefore, the initial number of neurons in each hidden layer is 10 . The initial weight and bias or threshold values are both random numbers ranging between -1 and 1 . The terminal criterion for concluding training is 5000 iterations or a minimum error smaller than $10^{-6}$. These criteria prevented excessive convergence times when the system is constrained in local optimal solutions.

Figure 7 represents a comparison of the error convergence curves for the data processed by the HTAIDSNN and HTAIDSNN with HDA and PCA. The curves show that the HTAIDSNN method requires 22.73 seconds to complete 195 iterations of network training. The HDA method requires 14.97 seconds to complete 131 iterations of network training, whereas the PCA requires more time and training iterations, that is, 18.51 seconds and 163 iterations. It is clear that the 


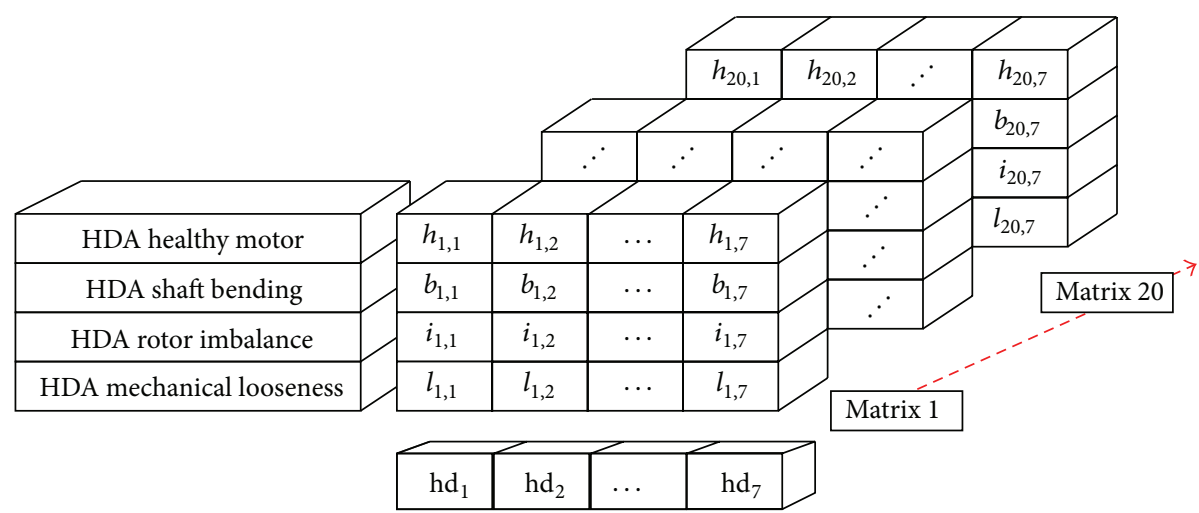

FIGURE 6: Neural network training matrix.

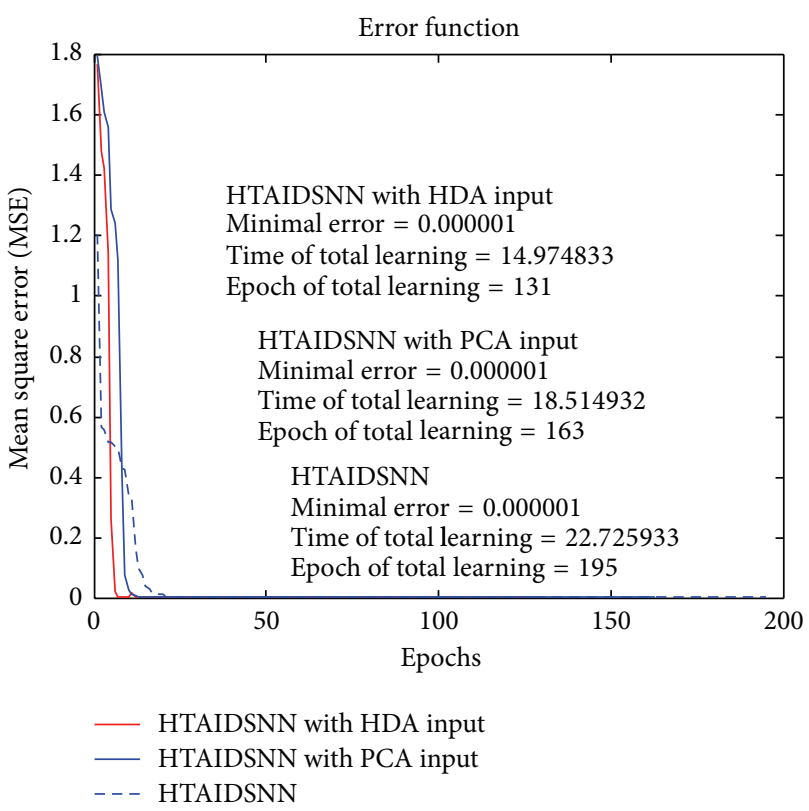

FIGURE 7: Convergence errors of HTAIDSNN with/without the addition of PCA and HDA.

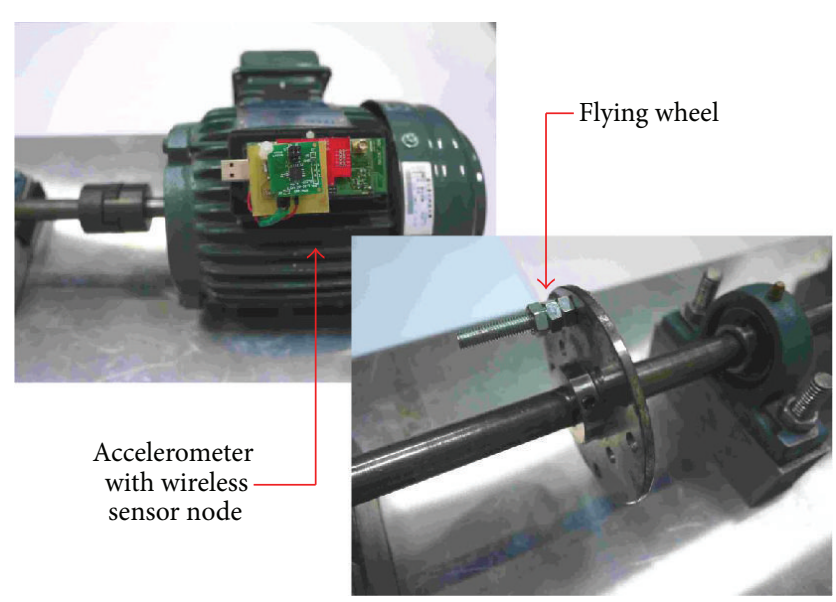

FIGURE 8: Equipment employed in the motor fault experiment.
TABLE 2: Classification statistics of the HTAIDSNN-based methods.

\begin{tabular}{lccc}
\hline $\begin{array}{l}\text { Motor } \\
\text { condition }\end{array}$ & HTAIDSNN & $\begin{array}{c}\text { HTAIDSNN } \\
\text { with PCA }\end{array}$ & $\begin{array}{c}\text { HTAIDSNN } \\
\text { with HDA }\end{array}$ \\
\hline $\begin{array}{l}\text { Healthy } \\
\text { motor }\end{array}$ & $95.9 \%$ & $98.4 \%$ & $99.95 \%$ \\
$\begin{array}{l}\text { Shaft bending } \\
\text { Rotor }\end{array}$ & $94.0 \%$ & $96.3 \%$ & $98.12 \%$ \\
$\begin{array}{l}\text { imbalance } \\
\text { Mechanical }\end{array}$ & $92.2 \%$ & $98.1 \%$ & $98.92 \%$ \\
looseness & $98.2 \%$ & $99.5 \%$ & $99.89 \%$ \\
\hline
\end{tabular}

HTAIDSNN with HDA has superior convergence performance. The improvement of the convergence rate is mainly due to the specific classification information provided by HDA.

Table 2 shows the statistics of fault classification using the HTAIDSNN-based methods. From the table, the performance of the HTAIDSNN with HDA is superior to the other two methods.

\section{Implementation of a Fault Diagnostic System}

5.1. Description of Experimental Equipment. The equipment employed in this study to conduct the motor vibration experiment included a four-pole, one-hp, three-phase induction motor manufactured by the TECO Electric \& Machinery Company (Taiwan). An A7200-MA VFD (TECO Electric \& Machinery Company) was also installed to change the rotational speed, and the source frequency was $60 \mathrm{~Hz}$. Figure 8 shows the wireless sensor nodes that are attached to the motor in the motor vibration experiment. An MMA7260 three-axis accelerometer was installed on a wireless sensor node (employed for transmitting vibration data) to measure vibration displacement.

During the experiment, screw nuts were installed on the motor flying wheel as unbalanced weights to generate the vibration fault signals that had frequency characteristics similar to that of an imbalanced rotor or bent shaft. The screws on the motor equipment stand were adjusted to emulate 


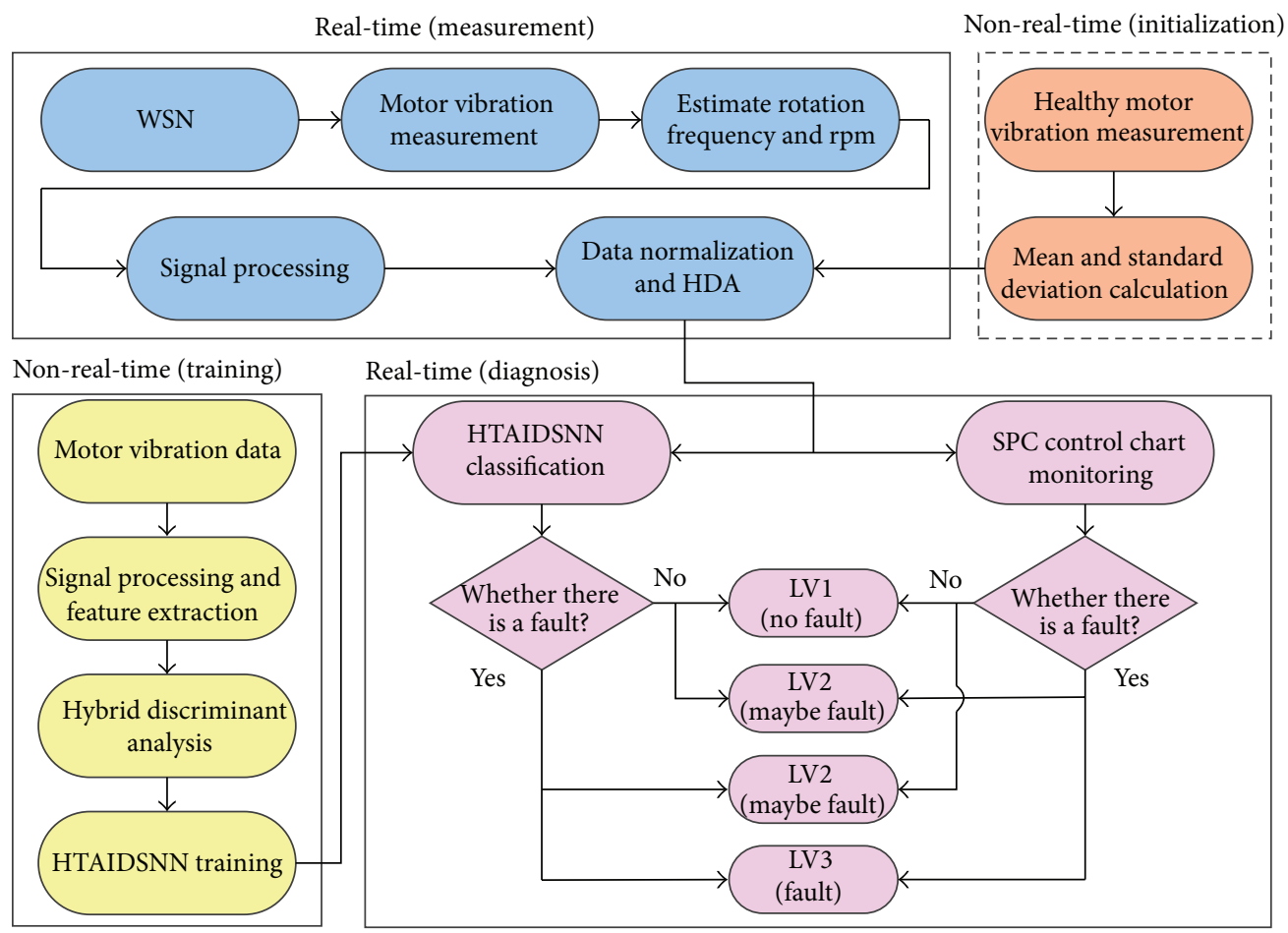

FIGURE 9: Flowchart of the intelligent speeding-varying rotary fault diagnostic system for motors.

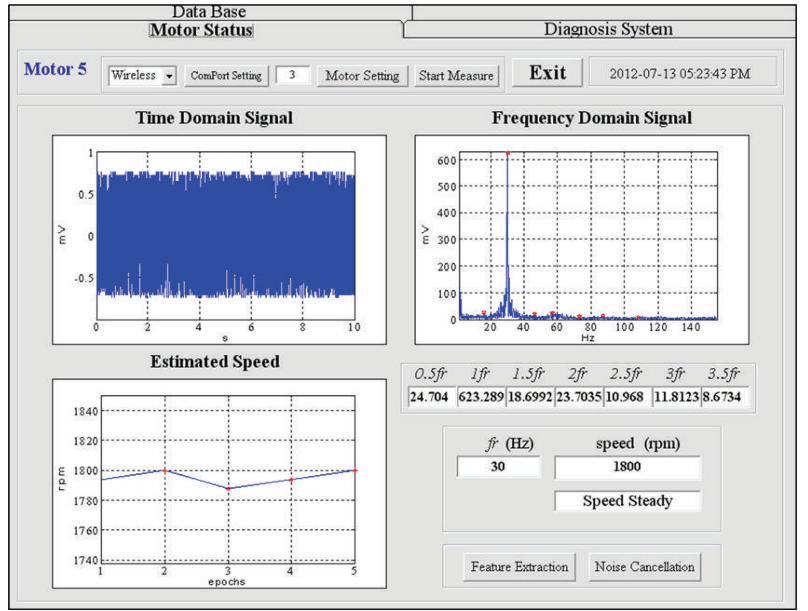

FIGURE 10: Status of an imbalanced rotor at a rotary frequency of $30 \mathrm{~Hz}$.

vibration fault signals resulting from loose components as shown in Figure 8.

5.2. Description of System Procedures. As depicted in Figure 9, this study initially retrieves the motor vibration signals using wireless sensor nodes and obtains the current rotary frequency through the sensorless rotational speed estimation method. Subsequently, the fault characteristics are extracted from the frequency-multiplier amplitudes of the rotary frequency, and the characteristic data are normalized. Finally, after HDA conversion, the neural network feeds the

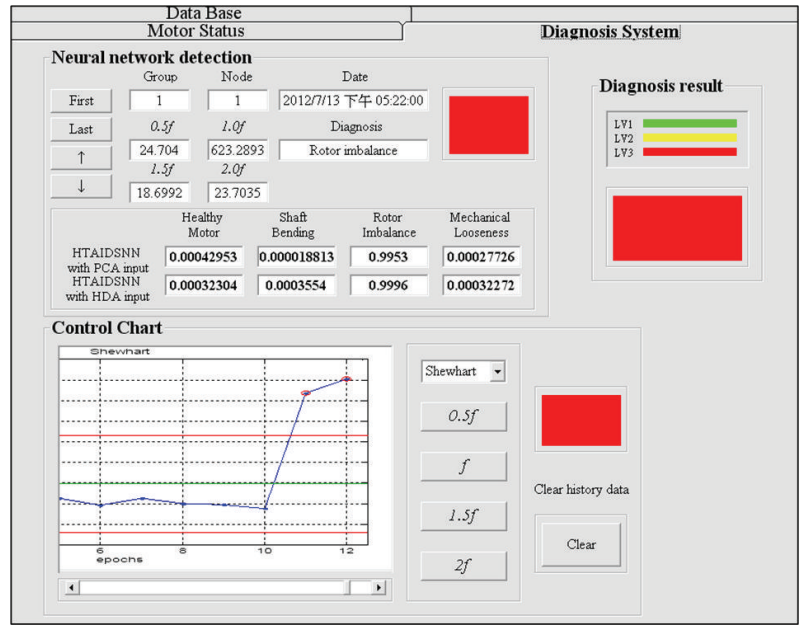

FIGURE 11: Diagnosis information for an imbalanced rotor at a rotary frequency of $30 \mathrm{~Hz}$.

compiled hybrid discriminant fault eigen-matrices into the HTAIDSNN for training of learning and memorizing for the relationships between the fault characteristics and fault classes.

In addition to the HTAIDSNN mentioned previously, control charts for stochastic process control (SPC) are included for fault diagnosis. The result of this combination is a dual core diagnostic system that employed parallel processing [21]. 


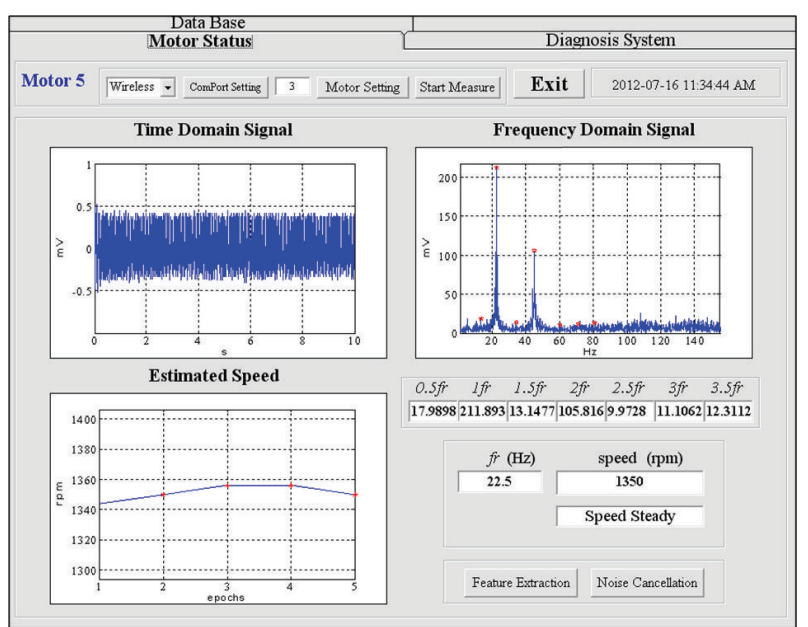

FIGURE 12: Status of a bent-shaft rotor at a rotary frequency of $22.5 \mathrm{~Hz}$.

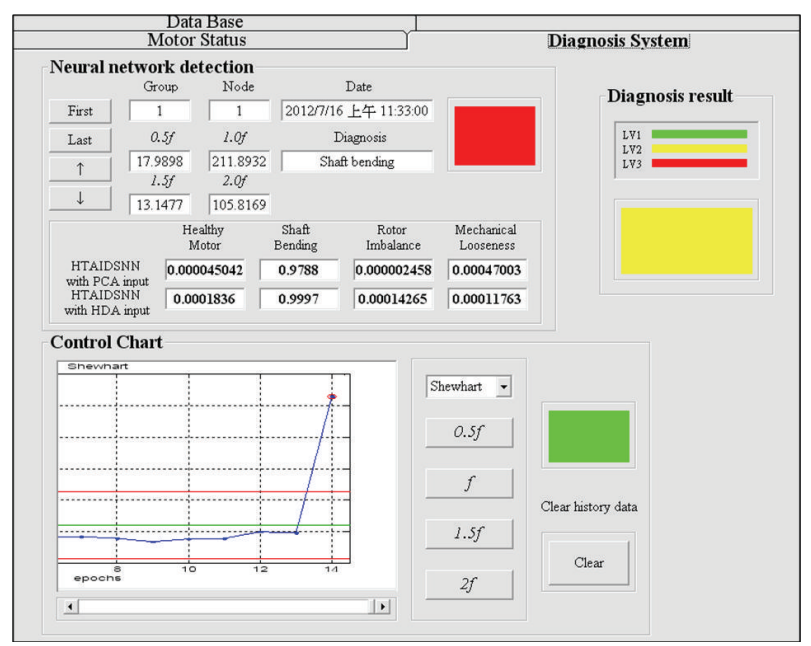

FIgURE 13: Diagnosis information for a bent-shaft rotor fault at a rotary frequency of $22.5 \mathrm{~Hz}$.

The SPC technique distinguishes between random outliers and abnormal statuses or states regarding the quality of monitored products to provide warnings concerning abnormal trends in the production process. Since the workplace is in general a noisy environment, the SPC charting used in this work is to monitor the shift and drift of the characteristic frequencies and rule out the abruptly environmental noise influence. The rule adopted in this paper is as follows: if there is only one point out of control limit, a warning is assigned. An abnormality in this study is defined as the situations in which two of three continuous or consecutive points are larger than control limit.

In the dual core diagnostic system, if the neural network and control chart are free from abnormalities, the system generates an LV1 signal to indicate the normal state. If either the neural network or the control chart generates an abnormal signal, an LV2 signal is generated to indicate that the motor may be faulty and require reconfirmation or stay alerted. If both are abnormal, an LV3 signal is generated to indicate that the motor is faulty and should be immediately repaired by technicians.

5.3. Description of the Human-Machine Interface. The human-machine interface designed in this study primarily includes a motor diagnostic system. Figure 10 shows the motor monitoring interface designed for the imbalanced rotor experiment conducted at a rotary frequency of $30 \mathrm{~Hz}$. This interface comprises a time-domain graph, a spectrogram, and a rotational speed estimation graph for the vibration signal. The frequency spectrum displays the peaks of the recent vibration signals at $0.5 f_{r}, 1 f_{r}, 1.5 f_{r}, 2 f_{r}$, $2.5 f_{r}, 3 f_{r}$, and $3.5 f_{r}$. Finally, the speed estimation graph displays the five most recent rotational speeds. The tolerable rotational speed change is $50 \mathrm{rpm}$. If the change of speed is larger than $50 \mathrm{rpm}$, the rotational frequency and speed are recalculated accordingly. To exclude the influences of noise or disturbance, the changes on the rotational speed are only displayed after the occurrence of the changes had been verified.

Figure 11 shows the interface designed for the diagnostic system used in the imbalanced rotor experiment conducted at a rotary frequency of $30 \mathrm{~Hz}$. This interface includes a neural network detection and Shewhart control chart. Both the neural network and the control chart in the figure detect an abnormality, and the overall diagnosis is marked in red (LV3). Figure 12 shows vibration signal, frequency spectrum, and the estimated rotational speed of a bent-shaft motor. Figure 13 indicates the diagnosis information of the motor. In the figure, the neural network detects an abnormality, and the Shewhart chart points out that there is a point out of control limit. By the rule defined in this work, this situation is acceptable unless the consecutive point is also out of control limit. Consequently, the overall diagnosis is classified as LV2 marked in yellow.

\section{Conclusion}

This study proposes a novel intelligent diagnostic system for speed-varying motors, which improves the convergence rate of traditional dynamic structural neural network training by introducing the terminal attractor and the hybrid discriminant analysis. The implemented system integrates the required functions: sensorless speed estimation, neural network-based diagnosis, and SPC charting, with a humanmachine interface to perform rotary fault diagnosis. The simulation results indicate that the proposed system is equipped with quick convergence rate for neural network training. Also, motor rotary faults are detected effectively by the developed diagnostic function and statistics process control chart.

\section{Conflict of Interests}

The authors declare that there is no conflict of interests regarding the publication of this paper. 


\section{Acknowledgment}

This work was supported financially in part by the National Science Council, Taiwan, under Grant no. NSC102-2221-E027-082.

\section{References}

[1] S. Nandi, H. A. Toliyat, and X. Li, "Condition monitoring and fault diagnosis of electrical motors-a review," IEEE Transactions on Energy Conversion, vol. 20, no. 4, pp. 719-729, 2005.

[2] S. A. Taher and M. Malekpour, "A novel technique for rotor bar failure detection in single-cage induction motor using FEM and MATLAB/SIMULINK," Mathematical Problems in Engineering, vol. 2011, Article ID 620689, 14 pages, 2011.

[3] H. Chen, Y. Lu, and L. Tu, "Fault identification of gearbox degradation with optimized wavelet neural network," Shock and Vibration, vol. 20, no. 2, pp. 247-262, 2013.

[4] H. Liu, J. Wang, and C. Lu, "Rolling bearing fault detection based on the teager energy operator and elman neural network," Mathematical Problems in Engineering, vol. 2013, Article ID 498385, 10 pages, 2013.

[5] W. Wang and O. A. Jianu, "A smart sensing unit for vibration measurement and monitoring," IEEE/ASME Transactions on Mechatronics, vol. 15, no. 1, pp. 70-78, 2010.

[6] D. C. Martinez, M. V. Rodriguez, A. G. Perez, R. A. O. Rios, and R. J. R. Troncoso, "Empirical mode decomposition and neural networks on FPGA for fault diagnosis in induction motors," The Scientific World Journal, vol. 2014, Article ID 908140, 17 pages, 2014.

[7] M. Iorgulescu, R. Beloiu, and M. O. Popescu, "Vibration monitoring for diagnosis of electrical equipment's faults," in Proceedings of the 12th International Conference on Optimization of Electrical and Electronic Equipment (OPTIM '10), pp. 493499, May 2010.

[8] A. Garcia-Perez, R. D. J. Romero-Troncoso, E. Cabal-Yepez, and R. A. Osornio-Rios, "The application of high-resolution spectral analysis for identifying multiple combined faults in induction motors," IEEE Transactions on Industrial Electronics, vol. 58, no. 5, pp. 2002-2010, 2011.

[9] K. Teotrakool, M. J. Devaney, and L. Eren, "Adjustable-speed drive bearing-fault detection via wavelet packet decomposition," IEEE Transactions on Instrumentation and Measurement, vol. 58, no. 8, pp. 2747-2754, 2009.

[10] H. Arabaci and O. Bilgin, "Effects of rotor faults in squirrel-cage induction motors on the torque-speed curve," in Proceedings of the 19th International Conference on Electrical Machines (ICEM '10), pp. 1-5, Rome, Italy, September 2010.

[11] M. Blodt, J. Regnier, M. Chabert, and J. Faucher, "Fault indicators for stator current based detection of torque oscillations in induction motors at variable speed using time-frequency analysis," in Proceedings of the 3rd IET Conference on Power Electronics, Machines and Drives, pp. 56-60, March 2006.

[12] H. Ocak and K. A. Loparo, "Estimation of the running speed and bearing defect frequencies of an induction motor from vibration data," Mechanical Systems and Signal Processing, vol. 18, no. 3, pp. 515-533, 2004.

[13] S. Guedidi, S. E. Zouzou, W. Laala, M. Sahraoui, and K. Yahia, "Broken bar fault diagnosis of induction motors using MCSA and neural network," in Proceedings of the 8th IEEE International Symposium on Diagnostics for Electrical Machines,
Power Electronics and Drives (SDEMPED '11), pp. 632-637, September 2011.

[14] F. M. C. Santos, I. N. D. Silva, and M. Suetake, "Neural network classifier for faults detection in induction motors," in Proceedings of the IEEE International Computer Applications Technology Conference, pp. 1-5, 2013.

[15] C. L. Chuang, Y. L. Lu, T. L. Huang, Y. T. Hsiao, and J. A. Jiang, "Recognition of multiple PQ disturbances using dynamic structure neural networks-part 1: theoretical introduction," in Proceedings of the IEEE/PES Transmission and Distribution Conference and Exhibition: Asia and Pacific, pp. 1-6, Dalian, China, August 2005.

[16] E. P. De Moura, C. R. Souto, A. A. Silva, and M. A. S. Irmão, "Evaluation of principal component analysis and neural network performance for bearing fault diagnosis from vibration signal processed by RS and DF analyses," Mechanical Systems and Signal Processing, vol. 25, no. 5, pp. 1765-1772, 2011.

[17] A. Abouhnik, R. G. Ibrahim, R. Shnibha, and A. Albarbar, "Novel approach to rotating machinery diagnostics based on principal component and residual matrix analysis," ISRN Mechanical Engineering, vol. 2012, Article ID 715893, 7 pages, 2012.

[18] B. Ayhan, M. Y. Chow, and M. H. Song, "Multiple discriminant analysis and neural-network-based monolith and partition fault-detection schemes for broken rotor bar in induction motors," IEEE Transactions on Industrial Electronics, vol. 53, no. 4, pp. 1298-1308, 2006.

[19] S. D. Wang and C. H. Hsu, "Terminal attractor learning algorithms for back propagation neural networks," in Proceedings of the IEEE International Joint Conference on Neural Networks (IJCNN '91), pp. 183-189, November 1991.

[20] J. Yu, Q. Tian, T. Rui, and T. S. Huang, "Integrating discriminant and descriptive information for dimension reduction and classification," IEEE Transactions on Circuits and Systems for Video Technology, vol. 17, no. 3, pp. 372-377, 2007.

[21] D. C. Montgomery, Introduction to Statistical Quality Control, John Wiley \& Son, New York, NY, USA, 6 edition, 2009. 


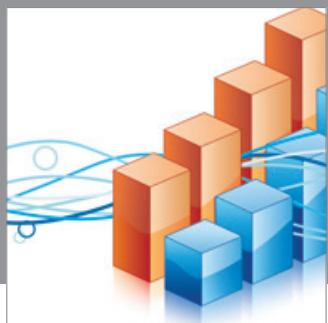

Advances in

Operations Research

mansans

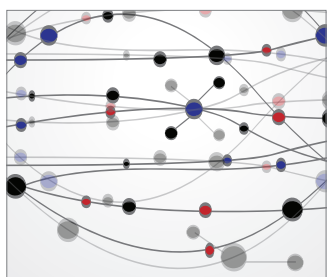

The Scientific World Journal
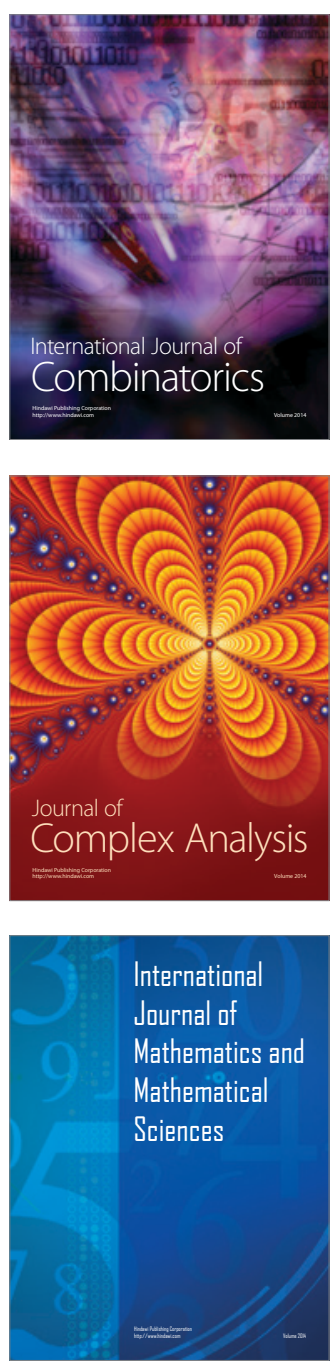
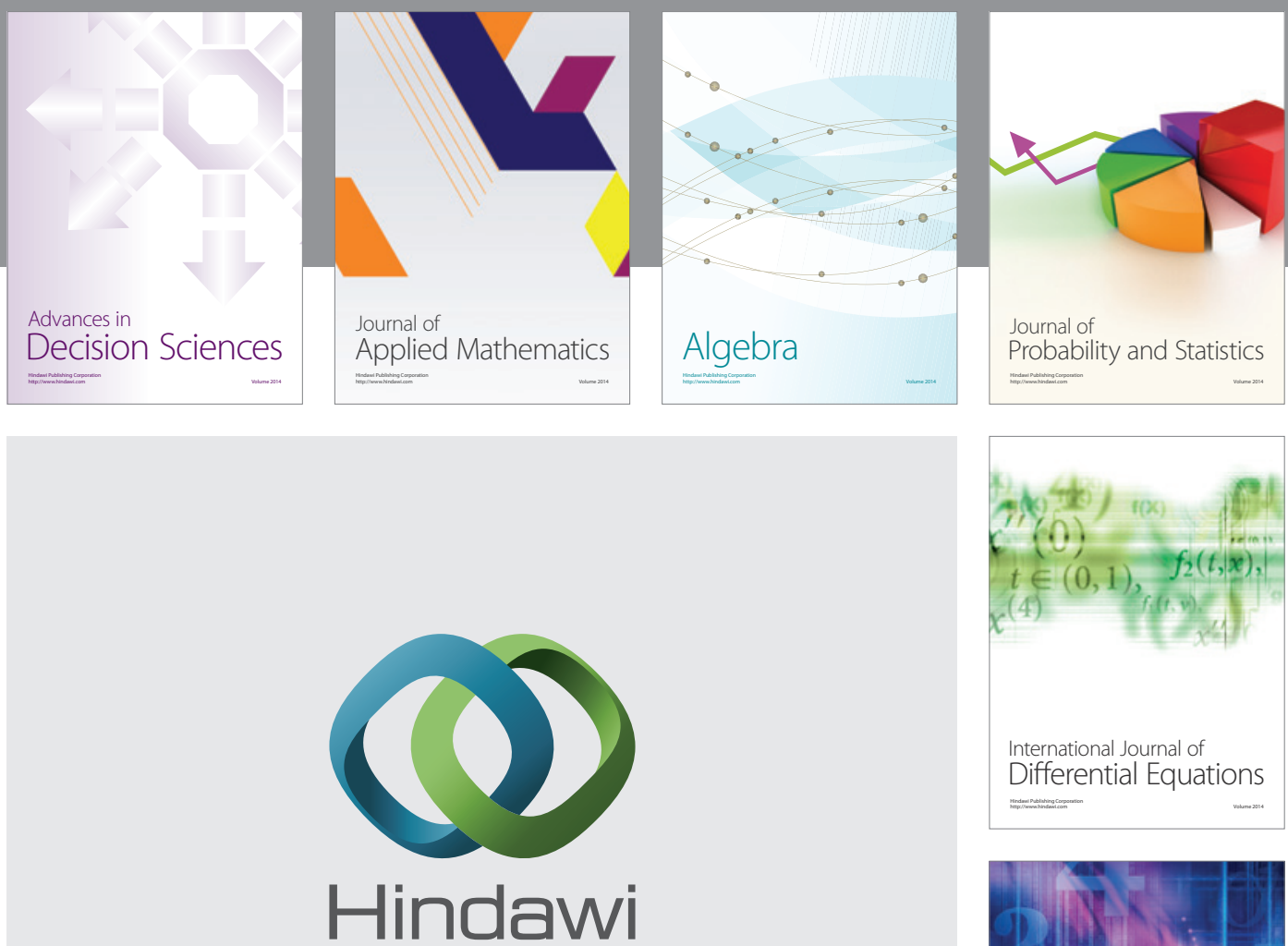

Submit your manuscripts at http://www.hindawi.com
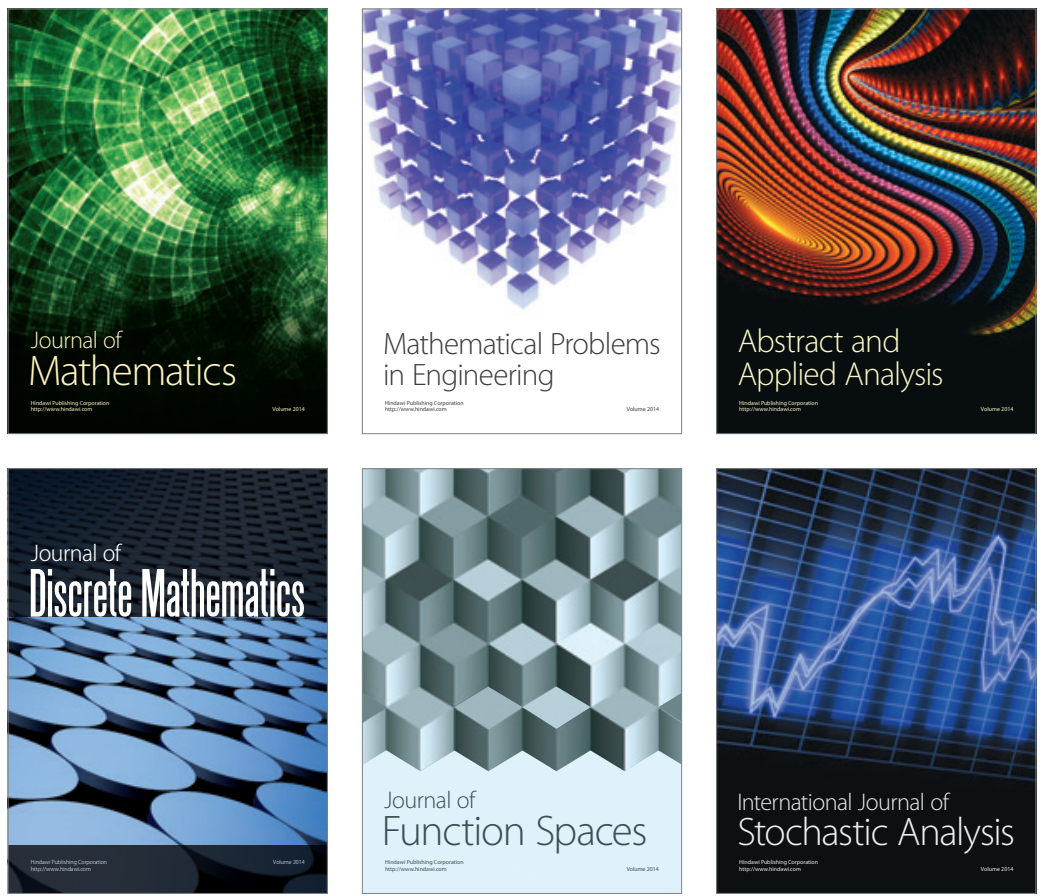

Journal of

Function Spaces

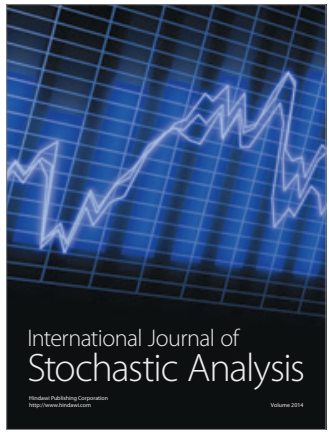

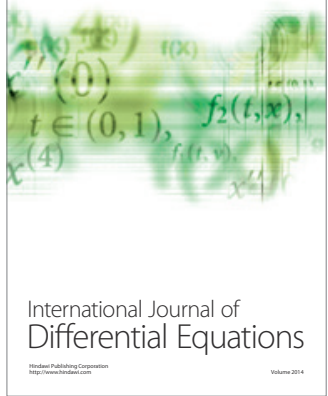
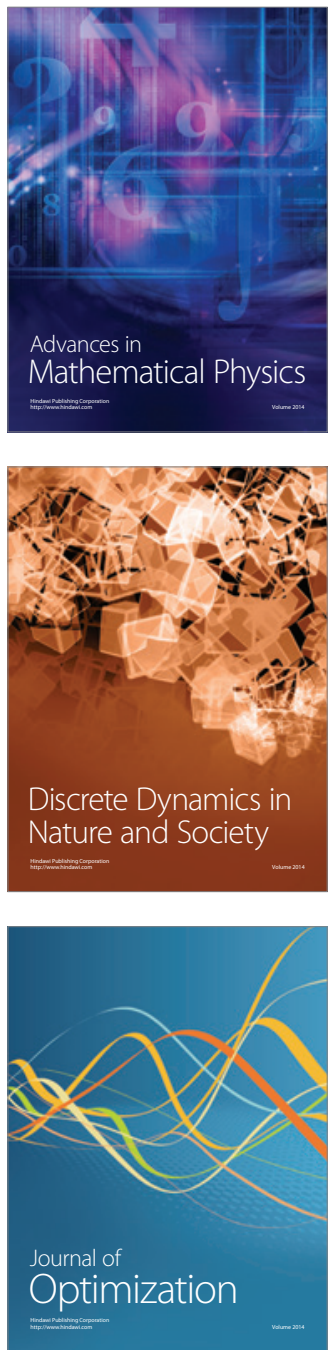